\title{
Cincuenta libros escolares de la Península de Yucatán, siglo XIX
}

\author{
Fifty School Books from the Yucatan Peninsula, 19th Century
}

\section{Martin Ramos Diaz ${ }^{1}$}

\begin{abstract}
Resumen
El propósito inicial de esta investigación fue elaborar un catálogo de libros escolares impresos en la Península de Yucatán durante el siglo XIX. En el proceso del registro detallado de libros para la confección del catálogo aparecieron vínculos frecuentes entre la élite letrada local y la dinámica actividad de impresión y reimpresión de libros escolares en la región. El catálogo regional de libros escolares decimonónicos va incluido al final del artículo, en forma de anexo: es un listado provisional que necesariamente seguirá creciendo con el aporte de los investigadores del sureste mexicano. Por ahora se trata de una nómina de medio centenar de libros escolares impresos en Campeche, Mérida y sus alrededores durante aquel siglo. La variedad de ramos educacionales - patente en los libros escolares registrados- es suma de los campos de conocimiento que la política pública de México designó como prioritarios para la educación en el inicio de la etapa independiente de la nación y posterior a las Leyes de Reforma (separación de Iglesia y Estado). La búsqueda y registro de libros escolares, impresos regionalmente, permitió documentar y escribir una narrativa sobre la relación entre élites letradas locales y relevancia de la educación pública decimonónica en ciudades como Campeche y Mérida.
\end{abstract}

\section{Palabras clave: libros escolares, Yucatán, educación siglo XIX}

\section{Abstract}

The initial purpose of this research was to develop a catalog of printed schoolbooks in the Yucatan Peninsula during the 19th century. Frequent links between the local literate elite and the dynamic activity of printing and reprinting schoolbooks in the region appeared in the process of detailed book registration. The regional catalog of nineteenth century schoolbooks appears at the end of this article in the form of an annex: it is a provisional list that will necessarily continue to grow with the

\footnotetext{
${ }^{1}$ Departamento de Humanidades, Universidad de Quintana Roo. Correo electrónico: martinramosdiaz@gmail.com
} 
contribution of researchers from southeast Mexico. For now, it is a list of fifty books printed in Campeche, Mérida and its surroundings during that century. The variety of educational branches - patented in the assembled school books - is the sum of the fields of knowledge that Mexico's public policy designated as priorities for education at the beginning of the independent stage of the nation and after the Reformation Laws (separation of Church and State). The search and registration of schoolbooks, printed regionally, allowed documenting and writing a narrative about the relationship between local literate elites and the relevance of nineteenth-century public education in cities like Campeche and Mérida.

\section{Keywords: school books, Yucatan, 19th century education}

\section{Introducción}

Hace medio siglo (1971), la Universidad de Alabama (University of Alabama) emprendió el proyecto de microfilmar gran cantidad de libros, revistas y documentos históricos de Yucatán. El resultado, fueron más de 120 rollos de microfilme que contienen la información almacenada por décadas en distintas instituciones culturales de Mérida. Esta colección yucateca constituye ahora el mayor registro de libros, diarios y revistas impresos en la región en el siglo XIX (Bingham, 1972: 1-100). Aunque en los últimos cincuenta años, los originales de algunos de esos documentos históricos desaparecieron de su repositorio institucional en el sureste mexicano, siempre es posible consultar la copia microfilmada.

La colección yucateca rescatada en microfilme fue la principal base documental usada en las siguientes páginas. La gran mayoría de los originales registrados en el anexo, al final de este artículo, fueron consultados en distintos veranos dedicados a trabajo de archivo en Mérida, Campeche, La Habana, Nueva Orleans, Austin, Madrid, Sevilla y, por supuesto, la Ciudad de México.

Por otra parte, la biblioteca latinoamericana de Tulane University cumplirá en 2024, una centuria de reunir colecciones bibliográficas, varias de las cuales provienen de Centroamérica (incluyendo a Yucatán), en donde generaciones de sus arqueólogos han trabajado por décadas. Los fondos bibliográficos de la casi centenaria biblioteca latinoamericana son indispensables para acercarse al panorama de los libros escolares decimonónicos impresos en Campeche, Mérida y sus alrededores. Igualmente, son imprescindibles las colecciones documentales de la Universidad de Texas (University of Texas) sobre los hacendados en Yucatán (Arrigunaga Family Collection) y, sobre los funcionarios gubernamentales del gobierno central mexicano desplegados temporalmente en la región (José Salazar Ilarregui Papers). En la documentación privada y comercial de hacendados del sureste de México y funcionarios del siglo XIX, siempre es posible encontrar catálogos de bibliotecas personales, descripciones de libros comprados en Europa, Estados Unidos y Cuba, como sucede en los casos Arrigunaga e llarregui. 
De los catálogos de libros de particulares (hacendados, funcionarios, sacerdotes, intelectuales) como de las librerías e imprentas de la región en el siglo XIX, se deduce que los libros escolares (impresos o no en la región) son un conjunto acotado, mínimo; parte un universo mayor de los libros que circularon en el sureste mexicano durante el siglo XIX. Las páginas que siguen detallan algunos ejemplares de ese acotado grupo de libros para escuelas en una región bifronteriza de México (frontera con el Caribe y con Centroamérica). Nuestra breve valoración incluye principalmente a los libros escolares que fue posible consultar en su totalidad y que nos parecieron centrales en la socialización de la cultura letrada de la región en el siglo XIX.

En la valoración de libros escolares del pasado, procuramos no olvidar que el conjunto amplio -libros de toda temática convergiendo en una misma región- son fuente, contexto y telón cultural de fondo, de los libros utilizados en la escuela. La narrativa de las páginas siguientes expone los vínculos encontrados, no siempre visibles, entre la edición local de libros escolares y la élite letrada regional. En algunas secciones fue inevitable vertebrar párrafos biográficos cortos con el modesto propósito original de elaborar un catálogo. El pasado educativo de una región bifronteriza de México impone una variable adicional que, en este texto, quedará esbozada y que dejamos para otra ocasión: la extranjería y su decisiva participación en proyectos educativos locales.

\section{Aficionado a las matemáticas: libros de aritmética}

La Escuela de Matemáticas y Náutica de Campeche fue el proyecto escolar más ambicioso de la ciudad al inicio del siglo XIX. La extendida y desigual historia de esta casa de estudios, uno de los primeros colegios navales del país, ha sido recuperada tanto por historiadores regionales (Lanz, 2008: 196-204) como por historiadores de la milicia y marina en México (Heroica, 1980: 16-18). Lo que la historiografía regional no ha recuperado, es la relación de alumnos y profesores de la escuela de matemáticas y navegación con la enseñanza de aritmética en la educación elemental de Campeche y Mérida.

No es fortuito encontrar, entre los docentes de la Escuela de Matemáticas y Náutica de Campeche, a autores de libros de aritmética para niños en una época en que los pilotos navales dependían tanto del conocimiento práctico del cayerío caribeño (islas y costas) como del conocimiento abstractos de temas avanzados de algebra, aritmética y geometría. Tampoco resulta sorprendente encontrar a exalumnos, pilotos campechanos de barcos mercantes que, mientras permanecían en tierra, dedicaban parte de su tiempo a enseñar operaciones básicas de aritmética a párvulos y matemáticas avanzadas a jóvenes de la región. Los Celarain, una familia de navegantes y curas, es uno de esos grupos familiares locales ligados a la Escuela de Matemáticas y Náutica de Campeche y luego al Liceo Carmelita. 
El Faro Celarain señala, actualmente, la parte sur de la Isla de Cozumel. El nombre de aquella punta de isla fue, durante mucho tiempo, Punta Celarain. Los isleños y las etiquetas sobre mapas, de tanto abreviar punta Celarain, faro Celarain, olvidaron a Juan Pablo Celarain: la propia enciclopedia alfabética de Yucatán (Duch, 1998) no registra entrada alguna para Juan Pablo Celarain, piloto formado en la Escuela de Matemática y Náutica de Campeche, destacado miembro de la marina mercante, jefe político de la Isla del Carmen y presidente de la junta directiva del Liceo Carmelita en 1858 (este liceo es el origen de la actual Universidad Autónoma del Carmen). Sin embargo, en los periódicos de la época quedaron indicios de la ruta marítima que frecuentó este piloto de la primera generación de la escuela naval campechana: la ruta Campeche-Veracruz-Campeche.

El periódico El Fénix del 11 de febrero de 1834, en su sección de entradas y salidas de naves en el puerto de Veracruz, registró al "bergantín nacional Sancho Panza, su capitán C. Juan Pablo Celarain, para Campeche". En la edición del martes 27 de septiembre de 1836, El Diario del Gobierno de la República Mexicana publicó en su sección de entradas y salidas de naves en el puerto de Campeche que la goleta nacional "Correo de Campeche cargó un poco de anís: su capitán, ciudadano Juan Pablo Celarain". Así, vemos que el inicio de la carrera naval de Juan Pablo en la promisoria ruta marítima Campeche-Veracruz-Campeche lo mantuvo en el área. Sabemos que hizo cabotaje por décadas en el Golfo de México y en el Mar Caribe por su autoría en la copia de un mapa de la península en 1841 (Reed, 2001: 64) y por la redacción del apéndice de una guía para navegantes y viajeros (Itinerarios, 1851) en la que describe los puntos notables de la costa y las vigías existentes en el litoral yucateco. ¿Es el joven piloto, o su hermano clérigo, autor de uno de los primeros manuales de aritmética para niñas en Campeche?

Su hermano, el cura José María Celarain, más conocido por fundar un asilo en Mérida, fue también profesor en el Liceo Carmelita. No obstante, el hijo de Juan Pablo, matemático como su padre y al mismo tiempo clérigo como su tío, ocupó la cátedra de Matemática Superior, Cosmografía y Pilotaje en la Escuela Náutica de Campeche a mediados del siglo XIX. La base de esa cátedra, a cargo del cura Rafael Celarain, fue el libro de texto de Gabriel Císcar y Císcar, destacado matemático español y gobernador de Cartagena, y cuyos libros escolares de instrucción marinera eran usados en la propia escuela naval de Cartagena. Retornando a Campeche y a las primeras décadas del siglo XIX, podemos verificar que de una imprenta de ese puerto (la de Lucas Guzmán) salió un temprano texto de aritmética: es el breve apéndice de matemáticas incluido en el Libro primero o nuevo arte de enseñar a leer teórica o prácticamente a las niñas dispuesto por un amigo de ellas, publicado en 1832.

Otro caso, también poco documentado, es el de José Martín y Espinosa y el extenso libro de aritmética para niños, publicado en 1833. José Martín y Espinosa fue profesor de matemática superior de la escuela naval de Campeche (en 1832) y, brevemente, director de aquella escuela (1841-1842): él es, probablemente, el compilador del extenso libro de 
aritmética, impreso en Mérida por su hijo impresor. En ese libro inusualmente extenso, el anónimo compilador firma como "un aficionado a las matemáticas", Arismética numeral que para beneficio de los alumnos de primera educación y en vista de los mejores autores, dispuso un aficionado a las matemáticas en forma de diálogo. Cuaderno I que contiene la logística de los números enteros (1833). El medio centenar de páginas de este libro escolar de aritmética contrasta con la brevedad de los catones para lectura y escritura y las cartillas alfabetizadoras destinadas a las escuelas elementales de aquel siglo. La famosa imprenta de José Dolores Espinosa (González Calderón, 110-136: 2014), el hijo mayor de Martín y Espinosa, produjo la edición de 1833. A lo largo del siglo XIX, la imprenta de Espinosa Losa imprimía libros de texto que comúnmente se usaban en escuelas de la región. A diferencia de los ramos de escritura y lectura, la legislación de Yucatán no introducía, en el caso de la aritmética, autores específicos o libros escolares obligatorios. Por ello, las sucesivas ediciones de la Arismética numeral aparecieron en cuadernillos más breves, con título diferente y dieron relevancia a las operaciones más tortuosas para el escolar (como los quebrados).

Previo a la aritmética impresa por Espinosa hijo, el texto que localizamos es, como quedó anotado, la breve sección denominada "Ideas primarias de los números", en el Libro primero o nuevo arte de enseñar a leer teórica o prácticamente a las niñas dispuesto por un amigo de ellas (1832). El concepto de número y las clases de número son los temas que el manual aborda en la reducida sección de aritmética. Lo notable en este caso es que se trata de un libro escolar para niñas, por lo que surge la siguiente interrogante: ¿los conceptos culturales de la época sobre la enseñanza de las operaciones básicas de la matemática consideraban suficiente una breve sección en el libro escolar de las niñas y una más extensa en el de los niños?

Las ediciones posteriores de libros de texto para aritmética fueron como un ejercicio de adaptación. Es como si el impresor y los maestros aprendieran - sobre la marcha - a dosificar temas, ejercicios y cantidad de páginas. Esto porque en lo sucesivo, es decir, en los dos últimos tercios del siglo XIX, los autores colocaron menos páginas en los libros escolares de aritmética (alrededor de 15 páginas y no el medio centenar de la edición de 1833), así como títulos en las portadas que resaltaban los temas matemáticos fundamentales en la enseñanza escolar elemental. De la Imprenta de Espinosa salió también: Explicación de quebrados comunes y decimales, números denominados, reglas de proporción de compañias, aligación y falsa posición (1846). El paso de más de una década entre la aparición del libro de aritmética de 1833 y el de 1846 aligeró el libro inicial. La versión siguiente, no solo breve sino puntual en las operaciones matemáticas de la enseñanza escolar en la época, gozó de mayor aceptación. Debió venderse bien porque, en el último tercio del siglo XIX, todavía uno de los descendientes del impresor Espinosa reeditaba el libro escolar de aritmética según la edición de 1846. 


\section{Costumbres puras, modales finos, ocupaciones suaves: libros para niñas}

Uno de los libros escolares de dibujo más utilizado en las escuelas decimonónicas locales de niñas del último tercio de siglo XIX, fue el de Félix Ramos i Duarte, joven cubano exiliado en Mérida. El bifronterizo emplazamiento de la Península de Yucatán (frontera con el Caribe, y Centroamérica) se hace evidente en el frecuente registro de extranjería asociada a la educación y a los libros escolares en la región. A mediados del siglo XIX, Honorato Ignacio Magaloni, un educador italiano, dirigió el Liceo Carmelita en la primera etapa de aquella institución educativa campechana (1854-1856). Y, como ya vimos, José Martín y Espinosa, un piloto náutico español, dirigió la Escuela de Matemática y Náutica (1841-1842). El paso de Felix Ramos i Duarte por Mérida es parte de ese emplazamiento de Yucatán en una frontera doble, de mar y de tierra. El legado bibliográfico menos conocido de Ramos i Duarte es el libro escolar para señoritas sobre dibujo aplicado a costura, bordado y tapicería. Más conocida y estudiada es su obra lexicográfica, Diccionario de mejicanismos (1886). Sin embargo, aquí solo nos ocuparemos de su Elementos de dibujo lineal para señoritas (1879), impreso en Mérida.

Para el último tercio del siglo XIX, la más longeva familia de impresores en Yucatán, continuaba su valiosa labor editorial en la región. La tercera generación Espinosa, el apellido de editores y libreros locales que perduró a lo largo de casi todo el siglo, se mantenía al frente del negocio de edición de libros escolares en Mérida y Campeche. De la imprenta de Miguel Espinosa Rendón procede Elementos de dibujo lineal para señoritas. Obra aceptada oficialmente para testo de las escuelas municipales del estado (1879). El autor, Félix Ramos i Duarte, trabajó en distintas ciudades de la república mexicana como educador, iniciando por Mérida. Su obra publicada más significativa sobre nuestro país fue, como quedó anotado, de corte lexicográfico, el Diccionario de mejicanismos (1886), el cual fue un libro pionero en el tema. En Mérida, Ramos i Duarte construyó su reputación como profesor con el libro Elementos de dibujo lineal para señoritas, un texto que venía trabajando desde principio de la década de 1870.

El bordado y costura respaldados por las clases de dibujo era en las niñas, el equivalente a la buena caligrafía apoyada en la habilidad del dibujo en los niños. Animales, frutos y objetos en costuras y bordados corresponden a un trazo y un significado en el libro de Ramos i Duarte. Los cuellos, bolsas de camisa, fundas y manteles: los espacios para bordar fueron hojas de cuadernos diferentes para el trazo de una caligrafía de hilos y estambres. El libro escolar de Ramos es una sorprendente inmersión en el mudo de los tejidos y la pasamanería, los hilos y las telas. Es un paseo por todos aquellos materiales con los que el dibujo se traslada del plano del molde al relieve del bordado. La ley de escuelas en Yucatán incluyó desde 
mediados de aquella centuria la costura en blanco, el bordado y la economía doméstica en la educación de las niñas (Ley, 1862: 162). Los oficios que convenían a las niñas, "débiles y propensas a manifestar emociones", según Catecismo de economía doméstica (1890: 15-16), eran aquellos oficios que requerían más habilidad que fuerza. Artes que "entretengan, como el dibujo, la costura y el bordado" (Catecismo, 1890: 17).

Para el amplio sector de familias pobres de la península de Yucatán y para las mujeres de cualquier clase social, se asignaba una ruta educativa muy parecida: ellas y los pobres quedaban fuera de la educación superior. Los libros escolares para las niñas enseñaban en sus lecciones que la educación era distinta para las clases socioeconómicas como para el género. Dice el Catecismo de economía doméstica (1890) que la educación primaria, así como los oficios y artes, era la educación "que pueden adquirir los pobres con más facilidad" (1890:16). La educación primaria consistía en leer, escribir y contar en un nivel básico. Los oficios y artes habilitaban en ocupaciones sobre las que funcionaban el comercio, la agricultura y la elaboración de productos en la región. La diferenciación socioeconómica para adquirir educación no parece menos flexible que la diferenciación por sexo: "La educación de los dos sexos se diferencia en mucha parte, porque la mujer debe tener costumbres más puras, modales más finos y ocupaciones más suaves que el hombre."(Catecismo, 1890: 15). Los dos temas dominantes en el Catecismo (1890): gobierno de la casa y conceptos útiles para la economía del hogar resumen el destino educativo decimonónico para las niñas.

Salida de la imprenta de Gamboa Guzmán en Mérida, el libro escolar de las nuevas Lecciones de economía doméstica para uso de las escuelas primarias (1895) son una adaptación local del libro de la maestra española Luciana Casilda Monreal, Cartilla de higiene y economía doméstica para uso de las escuelas de niñas (1890), publicada en Barcelona (imprenta de Pedro Ortega). Los detallados diálogos dedicados a los consejos de preparación y conservación de comidas y bebidas no pasan desapercibidos para el lector contemporáneo. El húmedo y caluroso trópico del sureste mexicano debió ser un reto cotidiano para el almacenamiento y conservación de la comida. Seguramente, los peninsulares de Yucatán apreciaron que, en los textos para niñas, se enseñara la manera de conservar pescados, "salándolos y en escabeche, que se guarden dentro de barriles y latas"; y los huevos, metiéndolos "entre ceniza y arena, y también recubriéndolos con una capa de cal" (Lecciones, 1895: 9).

El precedente español de las nuevas Lecciones de economía doméstica (1895) se percibe en algunos diálogos como el de la bebida: advierte el texto que las más perjudiciales son el habanero, el coñac, el verdín y el vino falsificado. La tropicalización de las lecciones podría hacernos suponer que el libro escolar tiene una autoría regional: "¿Qué desayuno se usa para casi todo Yucatán? El chocolate" (Lecciones, 1895: 12). Pero, como dijimos, es una derivación del libro escolar de doña Luciana Casilda Monreal.

La preparación de aguas frescas, helado de agua y leche (mantecados) son recetas imperdibles, y tienen un mayor perfil de pertenecer a la historia culinaria local. La tablilla de 
chocolate - pasta compuesta de cacao, azúcar, canela y en ocasiones vainilla- diluida en agua hirviendo, batida con la ayuda de un molinillo, es la conocida receta de las bebidas calientes. Menos conocidas en otras regiones de México son las bebidas destinadas a mitigar el calor del sureste: "¿Qué refrescos de maíz se usan en Yucatán? El pozol o keyén; el pinole o kah y el sacá, preparado con miel o azúcar" (Lecciones, 1895: 13). Al catálogo de ingredientes para hacer horchatas - almendras, arroz, pepitas de melón- y frutos locales para helados (guanábana, mamey, piña), lo acompaña un catálogo extendido de obligaciones femeninas como lavar, planchar, trabajos de costura, jardinería, limpieza, atención de las visitas, aseo de vajillas, entre otros (Lecciones, 1895: 26).

\section{El espíritu del trazo: libros de caligrafía}

Sobra decir que los libros de caligrafía impresos regionalmente durante la primera mitad del XIX fueron adaptaciones de los manuales españoles. Los autores locales figuran hasta los años finales del siglo y particularmente uno de ellos, Arturo Palmero Alcocer, es difícil de historiar. El panorama en materia de enseñanza de la caligrafía va más allá de la mera narrativa de la publicación local de distintos libros de texto para la enseñanza de escritura a lo largo de dicho siglo. No solo fue la pluma metálica y la máquina de escribir lo que transformó la cultura letrada de la región en los años finiseculares: en el caso de Yucatán no hemos estudiado en qué medida la mayor disposición de litografías (mediados del siglo XIX), introducidas en libros, revistas y diarios de la época y, el posterior uso de la fotografía (introducida en Yucatán por viajeros, arqueólogos y exploradores), renovaron la voluntad de leer y aprender a escribir. Litografías y daguerrotipos en los libros escolares del siglo XIX parecen notables impulsores indirectos de la alfabetización en el sureste de México que aguardan ser estudiados. Lo mismo sucede con las adaptaciones locales de los manuales de caligrafía españoles y con la biografía de los calígrafos del sureste de México, que también esperan ser estudiados.

Los periódicos decimonónicos de Yucatán, más que la legislación educativa local de principios de siglo, recogen en su sección de avisos el método de enseñanza de caligrafía utilizado en la región. Un aviso comercial publicado en la Gaceta de Mérida, en el número 198 de la edición del 16 de febrero de 1825, informa que el maestro de primeras letras, Pablo Cristóbal Salazar, ofrece al público "enseñar con esactitud y equidad... los primeros rudimentos de leer y escribir por el método de Torcuato" (Aviso, 1825). El aviso en la Gaceta de Mérida, la legislación educativa yucateca de mediados de siglo y los libros de caligrafía impresos en la región registran una extendida presencia en el sureste mexicano de Torcuato Torio de la Riva, el conocido calígrafo español. ¿Y por qué no habría de ser así?, si el método de Torcuato fue una real cédula en el pasado novohispano: fechada el 31 de enero de 1800, la cual obligaba 
a los preceptores de primeras letras en la Nueva España a enseñar escritura con el manual de Torio de la Riva.

Torcuato fue para la enseñanza de la caligrafía en el siglo XIX lo que la pluma de ave y el papel a la escritura: una asociación imprescindible que duró más de cien años. Al declinar el siglo, cuando las plumas metálicas para escribir -compradas en La Habana por campechanos y yucatecos- terminaron por desplazar a las plumas de ave, Torcuato siguió presente en las lecciones de los libros yucatecos finiseculares de caligrafía. Como en el resto de México, el calígrafo español aparece constantemente en los registros locales. Se le nombra con familiaridad Torcuato, en el aviso en la Gaceta de Mérida de 1825; Torcuato de la Riva, como figura en la Ley sobre escuelas de Yucatán de 1841 y Torcuato Torio de la Riva en los manuales yucatecos de caligrafía (donde lo citan sin citarlo): como en el Compendio de caligrafía (1871) de Lázaro Pavia y en los Elementos de caligrafía (1887) de Arturo Palmero Alcocer.

Los preceptores de primeras letras arreglaron su "enseñanza en la lectura al método de D. Vicente Naharro y en la escritura al de D. Torcuato de la Riva", dice la legislación educativa de Yucatán (Ley sobre escuelas, 1841). Pero como se desprende del aviso periodístico de 1825 y de la cédula real de 1800, el método de Torcuato se usó desde la centuria anterior. El apéndice del Catón cristiano con ejemplos para uso de las escuelas, impreso en Mérida en 1830 por José Atanasio Ortiz, es un temprano manual escolar con Torcuato como apéndice. El pie de imprenta de este libro advierte que es un reimpreso. Y el extendido título del Catón cristiano anuncia: "Añadido con un ecselente método para escribir por reglas". Bien podemos decir que el apéndice sobre escritura de la edición de 1830 del Catón cristiano es uno de los antiguos manuales de caligrafía en la región.

La descripción del procedimiento para cortar la pluma y los detalles para colocar el papel sobre la mesa durante los ejercicios de escritura caligráfica, según el "Método para escribir por reglas" de 1830, puede resultar sorprendente para el lector contemporáneo, hijo del bolígrafo y de la escritura digital. Sin alejarnos de la realidad decimonónica rural de Campeche y Mérida, podemos afirmar que la escritura caligráfica comenzaba cuando el escolar, o sus padres, perseguían un ave de regular tamaño. Porque, para que la pluma de escribir fuera útil, debía reunir tres características: a) fácil de sostener entre los dedos, es decir gruesa y redonda; b) suficientemente firme, pero no demasiado, para resistir el tajo apropiado en la base. La abundancia de manchas a lo largo del cálamo de la pluma indicaba dureza y dificultad para conseguir el tajo apropiado, así que era preferible plumas de cálamo claro (indicio de que eran flexibles). La última condición: c) que la pluma cayera sin dificultad sobre la coyuntura del dedo índice y pulgar. En consecuencia, se debía arrancar del ala derecha del ave.

Suponemos que era cansado perseguir al veloz plumífero para fabricar el dispositivo de escritura. Sin embargo, el escolar rural disponía de algunos consejos para improvisar en ciertas etapas del proceso: si la pluma no era sacada del ala derecha, se podía quebrar el 
cálamo en varias secciones hasta acomodarla a la mano del escolar. Una vez que el ave dejaba arrebatarse la materia prima, el interesado sumergía el producto en agua. Más allá, mientras el escolar curaba los mordiscos recibidos del ganso, pato o gallina, el agua de cenote hacía su trabajo: ablandar la pluma. Después de una noche inmersa en el agua del recipiente dejado al sereno (fuera de casa, a la intemperie) quedaba lista para el paso siguiente. La parte complicada de la mañana posterior no era desechar el agua y secar la pluma con un paño limpio. Raspar una sección del cálamo con el lomo de un cuchillo para despejar la base sobre la que se sujetarían los pequeños dedos del futuro escribiente, tampoco representaba problema alguno. El problema podía comenzar al cortar la base del cálamo, en el corte de un ombligo semihorizontal, donde la tinta haría su trapecio; es en este paso en el que muchas plumas de ave se malograban.

El corte "para todo carácter, excepto el inglés, suele ser su grueso de un sétimo de alto de la letra" (Método, 1830: 83). Conseguir esta proporción requería práctica: dar un tajo equivalente a la séptima parte del alto de la letra que se trazaría, no siempre se lograba. Si se corta más delgada la pluma "sale de poco espíritu la letra" (Método, 1830: 84) y la lectura se vuelve difícil. Si se corta más gruesa, la escritura no resulta hermosa: un corte muy grueso no permite dar a cada letra la combinación de los trazos anchos, delgados y medios. Pero de los dos extremos, aconsejaba el manual, mejor es usarla poco menos gruesa que exceder la proporción.

La posición del papel colocado en la mesa para los ejercicios caligráficos podría facilitar o entorpecer el deslizamiento de la pluma. La rigidez o la docilidad de brazo y mano determinaban el resultado caligráfico. Una escritura bien alineada no se trazaba sin la correcta posición del papel y sin el brazo y mano dócil. La hoja para la escritura debía colocarse en una posición media entre la horizontal y la vertical, como una prolongación de la postura del brazo. Oblicua la mano para escribir, oblicua la hoja del cuaderno de escritura: en un modo en que el compás del movimiento de la mano y brazo, ancladas en el codo descansado al borde de la mesa, se deslizara con igual soltura del inicio del renglón al final de éste. Esta posición conseguía que la primera y la última letra produjeran renglones alineados, en una época en la que el papel de escritura no era rayado sino un páramo blanco-amarillento.

La escritura era cálculo puro en los trazos de la caligrafía decimonónica: a ojo de buen escolar. Calcular la proporción de la letra, como calcular el imperceptible movimiento de la mano hacía el medio renglón mientras se escribía, evitaba la asimetría en la letra y la curva en el alineamiento de renglones (Método, 1830: 80-82). Nunca sabremos del agobio que producía en maestros y escolares los incontables detalles de la escritura caligráfica. La posición de la pluma, dice el Método de 1830, no debía variar durante el acto de escritura: la sección alta del instrumento, donde el cálamo adelgaza, caía en dirección de la coyuntura del dedo índice. La pluma: ni moverse, ni oprimirse entre los dedos, ni apretarse contra el papel. En la atmósfera tan preceptiva de la caligrafía, cada dedo tenía una obligación: el más pequeño, 
costado del meñique sobre la superficie, ayudaba a mover suavemente la mano en las oscilaciones de la escritura; mientras el pulgar servía para subir la pluma, el índice la bajaba, y los dedos medios eran soporte. Abrazar la pluma con los dedos correctos, sin despegarla del punto donde se inicia un trazo de letra larga (como la f) y llegar al punto final (Método, 1830: 76-80).

Si algo abunda en la península de Yucatán es luminosidad, cielos sin nubes durante muchos días del año, y sol que raja la piedra de los caminos que conducen a Campeche o Mérida. "Que haya luz bastante, mesa firma y asiento proporcionado" (Método, 1830: 74), pide el manual de caligrafía de 1830: estamos seguros de que luz hubo, pero tenemos escasos datos del "asiento proporcionado" y mesas firmes en las escuelas de la región durante las primeras décadas del siglo XIX. Un asiento proporcionado permitía al escolar mantener los pies apoyados sobre la tierra, con lo que facilitaba el trabajo del brazo, mano y dedos en los ejercicios caligráficos. Detalles como la distancia entre el pecho y el borde de la mesa, posición izquierda y derecha del cuerpo mientras se escribía y ubicación de ambas piernas del aprendiz frente a la mesa de escritura, hacen suponer que el manual de caligrafía estaba escrito más para los maestros que para los escolares.

Al final del siglo XIX, en el ámbito de la escritura ganó terreno la pluma metálica que terminó por sustituir a la pluma de ave (y también las "plumillas de madera", traídas del Puerto de Veracruz, se hicieron comunes). Luego se extendió el uso de las primeras máquinas de escribir. Las novedades finiseculares en Mérida y Campeche, en materia de caligrafía, fueron dos libros escolares: el Compendio de caligrafía (1871) de Lázaro Pavia y Elementos de caligrafía (1887) de Arturo Palmero Alcocer. El primer autor, más conocido por su actividad política en la vida pública de Yucatán que como calígrafo, recoge muchos elementos de Torio. El segundo, Palmero Alcocer, fue un médico yucateco menos conocido y más difícil de rastrear, quizá porque su actividad como profesor de caligrafía fue temporal. Sin embargo, su manual fue de uso obligatorio en algunas escuelas de la ciudad de México y en el estado de Yucatán, los lugares en que transitaba, ya como médico, ya como ocasional profesor de caligrafía.

\section{La superficie es nuestra: libros de geografía}

Los libros escolares para los nuevos ramos de la enseñanza después de las Leyes de Reforma, como en el caso de la geografía, tardaron en aparecer en las provincias rurales de México: estos no surgieron hasta la década de 1870, en el caso de Yucatán. Ni cartógrafos, ni paisajistas decimonónicos figuran en la península donde se unen las aguas del Golfo de México y el Mar Caribe. Con los dedos de una mano se cuentan los cartógrafos que dejaron obra importante para la región: Celarain, como ya vimos, dejó un mapa; otro más apareció, cuando la breve y poco conocida guerra Yucatán-Honduras Británica (1797-1798) obligó a levantar planos de una parte del cayerío (Spanish, 2020). En realidad, el cartógrafo local más famoso fue Joseph de Guelle (Antochiw, 2004), como el último intento de Yucatán por 
recuperar el actual territorio de Belice (1797-1798), pertenecen al pasado novohispano de la región. Además, paisajistas, esos artistas que hacen apología de las geografías regionales, tipo José María Velasco para el estado de México, son difíciles de hallar en el sureste mexicano para el siglo XIX. Hasta antes de la separación de la Iglesia y el Estado, el ramo de la geografía no aparece en la educación elemental. Como sucedió con el civismo y la economía doméstica para las niñas, la geografía fue un ramo de estudio contemplado en la educación pública mexicana después de las Leyes de Reforma, en la segunda mitad del siglo XIX. Un país tan joven como México, en aquel medio siglo, necesitaba urgentemente apropiarse de su geografía.

Aunque fue de las últimas regiones del México decimonónico en contar con su propio manual de geografía, los libros escolares de ese ramo transitaron por el mismo modelo que los del resto del país: la geografía era aprendizaje fundido con la historia. El caso es que el mismo año aparecieron dos libros de geografía impresos en la región: el dilatado volumen de Carrillo Ancona, Compendio de la historia de Yucatán; precedido del de su geografía y dispuesto en forma de lecciones para servir de texto a la enseñanza de ambos ramos en los establecimientos de instrucción primaria y secundaria (1871), y el modesto manual de José Correa Canto, Tratado elemental de la geografía de Yucatán (1871). Este último fue reditado varias veces hasta finalizar el siglo.

\section{El ceibo que te sirve de casa: libros de lectura}

Campeche y Mérida imprimieron sus propios libros escolares de lectura desde la década de 1830, alineados a la legislación local que prescribía el método para el ramo de leer. El esfuerzo por adaptar contenidos locales a las lecciones de leer por silabas es notorio particularmente en el último tercio del siglo XIX: "Huyendo del sol, viene el ganado a guarecerse bajo (la ceiba). Tú tienes mamá y papá, que te cuidan del sol, y casa que te sirve de ceiba" (Aznar Barbachano, 1898: 17).

El Catón cristiano con egemplos para uso de las escuelas. Con variedad de caracteres por su orden, y división de sílabas para que más fácilmente puedan aprender los niños. Añadido con un ecselente método para escribir por reglas, reimpreso en Mérida en 1830, recuerda la amplitud con que estos cuadernillos de contenido religioso fueron utilizados en todo México: como breves textos escolares para prácticas de lectura con los principiantes. En Campeche, el impresor Lucas Guzmán produjo el Libro primero o nuevo arte de enseñar a leer teórica y prácticamente a los niños dispuesto por un amigo de ellos (1832). La sección teórica, breve y destinada seguramente al maestro, definía y consignaba consonantes y vocales; aconsejaba sobre la articulación de las silabas y advertía de los casos de diptongo y triptongo. El Libro primero estaba compuesto por, esencialmente, 21 carteles de sílabas ordenadas alfabéticamente (omitiendo vocales) que replicaban el material del libro español de Vicente Naharro, 
Nuevo arte de enseñar a leer. Al final del siglo XIX es posible encontrar libros locales como el de Tomás Aznar Barbachano, Método práctico, gradual y progresivo para aprender y teórico-práctico para enseñar a leer bien (1898), que asimilan con provecho las discusiones de todo ese siglo sobre los métodos de enseñanza a los principiantes de lectura.

\section{Miscelánea: libros traducidos, diccionarios y ortografía, libros de moral y buenas costumbres}

Los libros escolares de Física, Botánica y Química, utilizados regionalmente en las escuelas superiores, fueron traducciones de conocidos hombres públicos de la localidad. Es el caso del curso elemental de Física de Auguste Pinaud, traducido e impreso en Campeche (1848) por Pablo García, prominente abogado y político que consiguió la creación del Estado de Campeche y Tomás Aznar, abogado, destacado educador y autor de varios libros escolares. El juez Serapio Carrillo, que tradujo un texto de moral e historia de la filosofía (Ética o moral, 1863), es un caso menos conocido de una época en la que se traducía localmente, con frecuencia textos franceses (Canto, 2011). Y también se pueden encontrar ejemplos de libros escolares donde no se menciona al traductor, pero se deduce que fueron impresos localmente, como los textos de botánica de Adrien-Henri de Jussieu (1873). En realidad, los vínculos de la élite letrada local con el trabajo de traducción de textos académicos para las escuelas de Campeche y Mérida aguardan ser evaluados.

La edición de enciclopedias parece una tradición en la localidad. La enciclopedia alfabética, Yucatán en el tiempo (seis tomos), es solo la más reciente expresión de ese género de libros. Pagada por el pueblo de Champotón, una imprenta de Campeche reimprimió la Biblioteca portátil popular (1839) destinada al apoyo educativo de los niños. Hasta donde pudimos verificar, de ese proyecto únicamente se publicó el primer volumen. Lo que llama la atención son las reimpresiones de libros prescriptivos de la lengua, como el caso del Prontuario de ortografía de la lengua castellana (1846), salido de una imprenta local de Mérida (Castillo y Compañía). No encontramos reimpresiones locales de gramáticas del español, pero sí del francés y del latín.

Hasta antes de las Leyes de Reforma, los libros locales de enseñanza de latín se nutrían de lecturas religiosas y textos morales transcritos de Cicerón. Véase las Advertencias y preceptos útiles para la clase de menores (1829), libro escolar salido de la imprenta a cargo de Lorenzo Seguí, un experimentado impresor establecido en Mérida, procedente de la Habana (González Calderón, 2014: 95). De su imprenta debieron salir varias ediciones del mismo libro, pero es notoria la edición de 1846 por su buena calidad tipográfica. Otro libro de enseñanza de latín para escolares avanzados, también impreso por Seguí: Construcción del libro cuarto para la clase de medianos (1846). 
Como los libros de geografía y economía doméstica, los libros escolares de moral, urbanidad y derecho se multiplicaron después de las Leyes de Reforma. Los editados localmente, adaptaciones de otros, fueron los siguientes: Principios de urbanidad para uso de la juventud, arreglados a los progresos de la actual civilización, seguida de una selección de fábulas en verso (1865), de Pío del Castillo; Tratado de deberes del hombre, para uso de los niños de las escuelas de primeras letras (1866) y Elementos de Moral (1879), de Tomás Aznar.

\section{Libros escolares en la región: los mismos y diferentes también}

En su juventud, Octavio Paz vivó en Mérida por algunos meses. El joven poeta capitalino vino como profesor de lenguaje a una escuela pública en 1937, durante el periodo presidencial de Lázaro Cárdenas. De esa experiencia escribió: "Aprendí algo que no he olvidado: México tiene otras tradiciones además de la del centro". De su estancia en el sureste de México-" un párpado de fiebre", dijo de la calurosa Mérida- quedó el libro Entre la piedra y la flor (poemas fechados en 1937, Mérida), extendido poema que, desde aquel año, anuncia los temas recurrentes en la poesía de Paz. De sus pensamientos sobre la región quedó el testimonio de su prosa: "Yucatán era México, pero también algo muy diferente".

De los libros escolares del siglo XIX podemos decir algo similar: son los mismos que en otras regiones de México, pero también son diferentes. Y, si recordamos el arribo de libros escolares a la región antes del siglo XIX, justificaremos la misma pregunta que en 1937 el joven Octavio Paz se hizo: “¿Qué tierra es esta?” (Paz, 1941: 5).

El anómalo camino por el que llegó el primer libro occidental a la región se resume a uno de esos casos en los que la cultura letrada del conquistador llega antes que las espadas y los cañones: Jerónimo de Aguilar, un náufrago español en las costas de Yucatán introdujo el primer libro europeo entre los mayas en 1511, mucho antes que los exploradores españoles arribaran a las costas (Hernández en 1517, Grijalba en 1518 y Cortés en 1519). Aguilar conservó su libro de Horas todos los años que estuvo perdido y esclavizado entre los mayas (1511-1519), hasta que Cortés lo rescató en Cozumel (Díaz, 1977: 102-104). El de Aguilar fue el primer "libro escolar" en Yucatán: las imágenes y las oraciones del libro de Horas fueron probablemente objeto de diálogo entre el náufrago español y sus amos mayas a quienes sirvió, sobre todo en la época en la que Aguilar habló con fluidez el maya.

Hoy sabemos por la dramática crónica de Fray Tomás de Torre que una parte de la biblioteca de Bartolomé de las Casas permanece en el fondo de la Laguna de Términos (Campeche). Como casi siempre, los religiosos dominicos y el obispo cruzaron sin problema el Atlántico y el Mar Caribe. Pero en la última sección de la travesía para llegar a Chiapas los sorprendió una tormenta en la Laguna de Términos: varios religiosos dominicos murieron ahogados y las cajas de libros terminaron en el agua. Del rescate: "nos trajeron una parte del 
Santo Tomás, que conocimos ser de Fray Miguel de Duarte" (Torre, 1945: 142). Los religiosos se quedaron sin parte de sus hermanos dominicos ahogados y sin la "consolación de libros ni cuadernos" (Torre, 1945: 147). Es decir, algunos libros escolares de los clérigos dominicos quedaron bajo el agua y nunca llegaron a su destino final en el obispado de Chiapas.

Los franciscanos abrieron las primeras escuelas de indios a mediados del siglo XVI (en Maní, Valladolid, Tekax) y los primeros estudios de gramática para indios y criollos en el área Yucatán-Guatemala a principios del siglo XVII (Mérida). Esa orden religiosa llegó y permaneció durante décadas de intenso trabajo evangelizador en la región. Los franciscanos del siglo XVI y XVII compilaron vocabularios de maya y ensayaron las primeras gramáticas de esa lengua. Diccionarios maya-español y esbozos de gramática de maya, compendiados por los religiosos, sirvieron de textos escolares a las sucesivas generaciones de religiosos que continuaron evangelizando en la península: Landa, Lizana y López de Cogolludo -por mencionar a tres conocidos franciscanos que dejaron obra escrita sobre Yucatán- aprendieron sus primeras lecciones de la lengua maya con la gramática y los compendios de sus antecesores.

La escuela de indios del convento de Maní fue notable bajo la dirección de un educador casi desconocido, Fray Juan de Herrera: "el primero que enseñó a cantar a los indios, el primero que les puso caracteres castellanos en las manos, y les hizo que leyesen, y escribiesen, y les enseñaba la doctrina en latín" (Lizana, 1988: 203). De la escuela de Maní egresó toda una generación de "nahuatlatos", traductores, escolares trilingües (maya, castellano, latín) que desempeñaron trabajos de escribanos, traductores y maestros de escuela en el último tercio del siglo XVI, como el caso de Gaspar Antonio Xiu.

El primer criollo de Yucatán en conseguir un doctorado en el siglo XVII, Pedro Sánchez de Aguilar, alumno del anciano Gaspar Antonio Xiu, hace un desapercibido ejercicio escolar neolatino con el título de su libro: Contra idolorum cultores (1892), un tratado breve en que Sánchez documenta y reflexiona sobre la persistente idolatría de los naturales de Yucatán. A mitad de camino, el título del libro de Sánchez entre latín y español, Contra idolorum cultores (1892), es solo evidencia menor de las prácticas escolares neolatinas comunes en el Yucatán del siglo XVII. Evidencia mayor de la práctica escolar neolatina en la región la encontramos a fray Bernardino de Valladolid, un religioso desconocido, quien trasladó del libro médico de Dioscórides, varios capítulos del latín al maya, entre 1648 y 1652. En todo caso, la práctica neolatina escolar en Yucatán durante el siglo XVII produjo libros escolares que todavía no evaluamos.

Propiciadas por Sánchez de Aguilar, las primeras incursiones de jesuitas a Yucatán para fundar un colegio de estudios superiores marcaron la siguiente etapa del arduo trabajo educativo de los franciscanos desarrollado en el siglo XVI. La mayor fuente para reconstruir los primeros colegios de jesuitas en Campeche y luego en Yucatán, en el siglo XVII, es Francisco Javier Alegre, un miembro de esa compañía religiosa que también vivió en Mérida por algún tiempo como profesor (Alegre, 1958, t 2: 307-309). Del Colegio de Mérida los jesuitas 
informaron en 1622 que: se "lee gramática a 70 estudiantes que, de esta provincia, de la de Tabasco y de la Habana se han recogido" (Zambrano, 1968, t. 8: 358). Los libros escolares de la etapa jesuita en la región y de todo el siglo XVII y XVIII tampoco los hemos investigado.

Los libros escolares del siglo XIX impresos en una región bifronteriza de México, reunidos aquí en un sencillo catálogo (ver anexo I), están a la espera del historiador de la cultura o del historiador de la educación. La pregunta formulada en 1937 por el joven poeta Paz, "¿qué tierra es esta?", no es una mera pregunta retórica. Para el historiador regional de la cultura impresa puede ser una pregunta productiva en la indagación de la historia del libro escolar en el sureste mexicano.

\section{Reflexiones sobre libros escolares decimonónicos impresos en Yucatán}

La ausencia de libros escolares de ciencia a lo largo del siglo XIX fue lo primero que notamos al finalizar el catálogo (la elaboración del catálogo que figura en el anexo I fue el propósito central de nuestra investigación). Por eso nos pareció relevante focalizar la atención en la Escuela de Matemáticas y Náutica de Campeche, institución que ha sido estudiada por distintos historiadores, y recuperar parte de la biografía de algunos de sus profesores que, como vimos, son los probables autores de los primeros libros locales de aritmética para niños en la mitad inicial del siglo XIX. El conjunto de libros escolares de aritmética y la nómina de probables autores locales que contribuyeron en la formación matemática de los niños en la región, apenas figuran en el catálogo: esa es razón suficiente para intentar un acercamiento a las aritméticas y a los aficionados a las matemáticas del primer apartado de la presente investigación.

Comprensiblemente, en los estudios mexicanos sobre la historia de la educación no es frecuente encontrar valoraciones, descripciones o panoramas de libros escolares de ciencia. Más difícil de encontrar son los esbozos biográficos de los anónimos mentores detrás de la formación matemática de los estudiantes mexicanos del siglo XIX. Es más usual entre los historiadores de la educación el abordaje de libros escolares asociados a los campos sociales y humanísticos. A la valiosa historiografía mexicana que da cuenta de los libros escolares en nuestro país le vendría bien detenerse con mayor frecuencia en el estudio de los libros escolares de ciencia, aunque sean tan básicos como los de aritmética para niños (ver sección 1). Para el caso de la región estudiada, existen campos por explorar, como las traducciones locales de libros franceses de Física, Química, Botánica, Mecánica, todos para uso escolar en el último tercio del siglo XIX (ver sección 6).

En ese camino, Antonio Viñao advierte de una precaución metodológica básica: "una cosa es la historia de los libros de texto y otra la de las disciplinas escolares" (Viñao, 2006: 14). En la sección uno conjeturamos sobre los autores-editores de los primeros libros regionales de aritmética para niños, insertamos párrafos biográficos que bien pueden agregar una 
tercera variable a la afirmación de Viñao: una cosa es la historia de los libros escolares, otra la de disciplinas escolares y una más la de editores-autores de libros escolares.

Los estudios de Roger Chartier sobre la cultura impresa, donde figuran sus espléndidas lecciones de arqueología de la edición (Chartier, 1997; 2016), son muy estimulantes para quien confecciona un catálogo regional de libros escolares decimonónicos. El conjunto de libros escolares para niñas en el siglo XIX es tan definido en sus contenidos y en su propósito que parece el ámbito propicio para indagar la procedencia de las lecciones, las variantes entre una edición y otra y la biografía de las autoras. A riesgo de cometer el error de suponer que solo con el catálogo de libros de un siglo, de un lugar específico, se puede hacer arqueología de la edición, los apuntes de la sección dos pretendieron asomarse a esa posibilidad.

El extendido uso de un libro escolar de dibujo para señoritas (1879) en las escuelas de Mérida y Campeche, preparado por un joven profesor cubano (Ramos i Duarte), exiliado en Yucatán, nos parecieron escenario perfecto para un ejercicio mínimo de arqueología de la edición. Un libro escolar tan cuidadosamente bien preparado, estructurado, con amplia y variada información, como el de Ramos i Duarte, requiere mucho tiempo de trabajo. Supusimos que el joven autor cubano avecindado en Mérida (luego en Veracruz y después en Ciudad de México) había preparado sus notas, muy probablemente, antes de llegar a Yucatán. Posiblemente en sus años de estudiante en una escuela de profesores en la Isla de Cuba. En todo caso, a principios de la década de 1870 el joven profesor ofrecía las clases de ampliadas se transformaron en el libro de 1879. Infortunadamente para el caso del libro escolar de Ramos i Duarte no encontramos la documentación o los manuscritos del autor, que permitieran documentar el origen de este manual escolar obligatorio en las escuelas públicas de la región en el último tercio del siglo XIX.

Mayores posibilidades para el ejercicio sobre génesis de los libros escolares locales las encontramos en otro libro regional para niñas, Lecciones de economía doméstica (1895). En este caso fue posible identificar sus semejanzas con un libro escolar español de economía doméstica editado cinco años antes en Barcelona. Fue práctica común, al menos en toda la primera mitad del siglo XIX, adaptar libros escolares españoles a la región. Los libros de lectura y escritura reimpresos en Yucatán en las décadas de 1820 a 1860 son buen ejemplo de ello (ver sección 5 y 3). En estos casos, los libros fueron una copia de sus similares españoles con un pie de imprenta regional. El ejercicio de arqueología de la edición de los libros regionales está pendiente para el caso de Yucatán. Con el catálogo que presentamos en el anexo I, los historiadores de la educación interesados en el asunto podrían avanzar.

Nos pareció importante destacar los libros de caligrafía de autores locales que fueron publicados en el último tercio del siglo (ver sección 3). El hecho de que personajes de la vida pública regional (Pavia) o médicos destacados (Palmero) figuren como autores de manuales de caligrafía permite apreciar la consolidación y rápida transformación del género de los libros escolares en la región. Los libros locales de caligrafía que en la primera mitad del siglo XX 
fueron reimpresiones de libros españoles, se transformaron cincuenta años después, en manuales estructurados en capítulos (y no en preguntas y respuestas), escritos por personas de la localidad (y no de autores españoles).

La tipología de libros escolares descrita por Choppin para el caso de Francia permite entender cómo el libro escolar adquirió identidad propia recientemente. En el caso de los libros locales de caligrafía pudimos ver este proceso y suscribir con el historiador francés que el concepto de libro escolar es históricamente reciente. Lo que sigue, para quienes intentamos registrar la cultura impresa en distintas regiones de México, es explicar (a partir de los libros registrados en el catálogo del anexo I) cómo la historia de la edición de libros escolares locales forma parte, "con toda seguridad" (Choppin, 2018: 6) de la historia regional.

\section{Bibliografía primaria (libros escolares)}

\section{Anexo I: libros escolares decimonónicos impresos en la Península de Yucatán}

Advertencias (1829). Advertencias y preceptos útiles para la clase de menores, Imprenta a cargo de Lorenzo Seguí, Mérida de Yucatán.

Advertencias (1846). Advertencias y preceptos útiles para la clase de menores. Corregidos y aumentados, Imprenta de José Dolores Espinosa, Mérida de Yucatán.

Advertencias (1855). Advertencias y preceptos útiles para la clase de menores. Corregidos y aumentados, Imprenta de José Dolores Espinosa, Mérida de Yucatán.

Análisis (1865). Análisis lógico de la proposición (castellana), Imprenta de Rafael Pedrera, Mérida de Yucatán. Arismética (1833). Arismética numeral que para beneficio de los alumnos de primera educación y en vista de los mejores autores, dispuso un aficionado a las matemáticas en forma de diálogo. Cuaderno I que contiene la logística de los números enteros, Imprenta de Espinosa, Mérida de Yucatán.

Aznar Barbachano, Tomás (1879). Elementos de moral, Imprenta de la Sociedad Tipográfica, Campeche.

Aznar Barbachano, Tomás (1898). Método práctico, gradual y progresivo para aprender y teórico-práctico para enseñar a leer bien, Imprenta de la Calle de América, Campeche.

Biblioteca (1839). Biblioteca portátil popular, Imprenta del Lucero, Campeche.

Carrillo y Ancona, Cresencio (1871). Compendio de la historia de Yucatán; precedido del de su geografía y dispuesto en forma de lecciones para servir de texto a la enseñanza de ambos ramos en los establecimientos de instrucción primaria y secundaria, Mérida de Yucatán.

Carrillo y Ancona, Crescencio; Molina, Audomaro (1887). Catecismo de la historia de Yucatán y compendio de la geografía de Yucatán, Librería Católica de Audomaro Molina y Compañía, Mérida.

Castillo, Pío del (1865). Principios de urbanidad para uso de la juventud, arreglados a los progresos de la actual civilización, seguida de una selección de fábulas en verso, Mérida.

Catecismo (1890). Catecismo de economía doméstica para el uso de las escuelas de niñas. Reimpreso con ligeras reformas. Librería de Espinosa, Mérida de Yucatán.

Catón (1830). Catón cristiano con egemplos para uso de las escuelas. Con variedad de caracteres por su orden, y división de silabas para que más fácilmente puedan aprender los niños. Añadido con un ecselente método para escribir por reglas, Oficina del Sol, reimpreso por José Atanasio Ortiz, Mérida de Yucatán. 
Compendio (1860). Compendio de la gramática y ortografía castellana, en forma de diálogo para la comodidad y uso de las escuelas de primera educación, Imprenta de José Dolores Espinosa, Mérida de Yucatán.

Construcción (1846). Construcción del libro cuarto para la clase de medianos, Imprenta de Lorenzo Seguí e hijos, Mérida de Yucatán.

Correa Canto, José (1871). Tratado elemental de la geografía de Yucatán, Mérida de Yucatán.

Correa Canto, José (1877). Tratado elemental de la geografía de Yucatán, Mérida de Yucatán.

Correa Canto, José (1898). Tratado elemental de la geografía de Yucatán, Mérida de Yucatán.

Diccionario (1866). Diccionario histórico de Yucatán. Tomo I. Mérida de Yucatán.

Elementos (1879). Elementos de las Cuatro Reglas Principales de la Aritmética para uso de niños que concurren a las escuelas de primera enseñanza, Imprenta y Librería de Miguel Espinosa Rendón, Mérida de Yucatán.

Estrada y Zenea, Ildefonso (1870). Diccionario de los niños, Mérida de Yucatán.

Ética o moral (1863). Instituciones filosóficas para uso de las escuelas de la Diócesis. Ética ó Moral, traducida al castellano por Serapio Carrillo, Mérida.

Explicación (1846). Explicación de quebrados comunes y decimales, números denominados, reglas de proporción de compañías, aligación y falsa posición, Oficina de José Dolores Espinosa, Mérida de Yucatán.

Explicación (1878). Explicación de quebrados comunes y decimales, números denominados, reglas de proporción de compañias, aligación y falsa posición, Imprenta y Librería de Miguel Espinosa Rendón, Mérida de Yucatán.

Irigoyen, Santiago (1872). Nociones de geometría, 2ª ed., Mérida de Yucatán.

Jussieu de, Adrien-Henri (1873). Resumen sucinto de los caracteres de las principales familias botánicas, Mérida.

Leal, Cecilio (1887). Tres leccioncitas de historia natural para niños, Mérida de Yucatán.

Lecciones (1883). Lecciones de agricultura arregladas para la escuela de instrucción primaria, Imprenta de la Revista de Mérida, Mérida de Yucatán.

Lecciones (1884). Lecciones de agricultura arregladas para la escuela de instrucción primaria, Imprenta de la Revista de Mérida, Mérida de Yucatán.

Lecciones (1895). Lecciones de economía doméstica para uso de las escuelas primarias. Imprenta Gamboa Guzmán, Mérida de Yucatán.

Libro (1832). Libro primero o nuevo arte de enseñar a leer teórica y prácticamente a los niños dispuesto por un amigo de ellos, Impreso por el ciudadano Lucas Guzmán, Campeche.

Libro (1876). Libro primario de los niños. Imprenta a cargo del padre Rafael Bolio, Izamal.

Máximas (1858). Máximas del hombre de bien o de la sabiduría y otras composiciones propias para instrucción de los niños, Mérida de Yucatán.

Nociones (1872). Nociones de geometría, extractadas de varios autores para el uso de las escuelas de instrucción primaria. Imprenta Literaria, Mérida de Yucatán.

Palmero Alcocer, Arturo (1887). Elementos de caligrafía, Imprenta Mercantil a cargo de José Gamboa Guzmán, Mérida de Yucatán.

Pavia, Lázaro (1871). Compendio de caligrafía general, Imprenta J. Gil Canto, Mérida de Yucatán.

Pequeño (1863). Pequeño catecismo de geografía. Arreglado y corregido con especialidad en las nociones preliminares y descripción de las dos Américas, Imprenta Carmelita, Campeche.

Pinaud, Auguste (1848). Programa de un curso elemental de física; traducido del francés por D. Pablo García y D. Tomás Aznar Barbachano, Campeche.

Pinto, Juan de Dios (1892). Tratado de dibujo lineal compuesto expresamente para uso de los artesanos que deben aprender sin maestro, Imprenta de la Revista Mérida, Mérida de Yucatán. 
Prontuario (1846). Prontuario de ortografía de la lengua castellana, dispuesto de real orden para el uso de las escuelas públicas, por la real Academia Española con arreglo al sistema adoptado en la novena edición de su diccionario, Mérida de Yucatán.

Ramos i Duarte, Félix (1879). Elementos de dibujo lineal para las señoritas. Obra aceptada oficialmente para testo de las escuelas municipales del Estado. Imprenta y Librería de Miguel Espinosa Rendón, Mérida de Yucatán.

Ripalda, Gerónimo de (1847). Catecismo y exposición breve de la doctrina cristiana por el padre maestro Gerónimo de Ripalda de la Compañía de Jesús traducida al idioma yucateco con unos afectos para socorrer a los moribundos por el M. R. P. Fr. Joaquín Ruz, Impreso por José D. Espinosa, Mérida de Yucatán.

Ripalda, Gerónimo de (1871). Catecismo de la doctrina cristiana por el padre Ripalda. Edición especial arreglada para la enseñanza de los niños en los templos, por la comisión respectiva, Imprenta de J. D. Espinosa e hijos, Mérida de Yucatán.

Ripalda, Gerónimo de (1892). Catecismo y exposición breve de la doctrina cristiana por el padre maestro Gerónimo de Ripalda de la Compañía de Jesús traducida al idioma yucateco con unos afectos para socorrer a los moribundos por el M. R. P. Fr. Joaquín Ruz, Mérida de Yucatán.

Sánchez de Aguilar, Pedro (1892). Informe contra idolorum cultores del obispado de Yucatán, México, Imprenta del Museo nacional.

Tabla (1861). Tabla aritmética y otras curiosidades útiles para la juventud, Imprenta de José Dolores Espinosa, Mérida de Yucatán.

Torre, Tomás de la (1945). Desde Salamanca, España, hasta Ciudad Real de Chiapa, 1544-1545, México, Editorial Central.

Tratado (1866). Tratado de deberes del hombre, para uso de los niños de las escuelas de primeras letras, Tipografía de E. Ancona, Mérida de Yucatán.

Tratado (1869). Tratado de deberes del hombre, para uso de los niños de las escuelas de primeras letras, Corregido y aumentado, Mérida de Yucatán.

Vidal Castillo, J. (1879). Compendio de la geografía de la Península de Yucatán, escrito para servir de texto en los establecimientos de instrucción primaria, Imprenta y Librería de Miguel Espinosa Rendón, Mérida de Yucatán.

Vidal Castillo, J. (1898). Compendio de geografía de Yucatán y Campeche y un apéndice sobre la colonia de Belize. Tipografía del Cobierno del Estado de Veracruz, Jalapa.

\section{Bibliografía complementaria}

Alegre, Francisco Javier (1958). Historia de la provincia de la Compañía de Jesús de Nueva España, Roma, Institum Historicum, $4 \mathrm{t}$.

Antochiw, Michel (2004), Alejandro Joseph de Gaulle. El primer cartógrafo de la Península de Yucatán, Campeche, Universidad Autónoma de Campeche.

Asociación Heroica Escuela Naval Militar (1980). Síntesis histórica de la Escuela Naval Militar, Ciudad de México, Archivo Histórico de la Secretaría de Marina.

Canto Mayén, Emilio Enrique (2011). Inmigración e influencia cultural de Francia en la región henequenera de Yucatán, 1860-1914, Ciudad de México, Instituto Mora, tesis de maestría.

Díaz del Castillo, Bernal (1977). Historia verdadera de la conquista de la Nueva España, México, Porrúa, tomo I.

González Calderón, Marcela (2014). La imprenta en la Península de Yucatán en el siglo XIX, Mérida, CIESAS (tesis doctoral). 
Duch, Juan (1998). Yucatán en el tiempo. Enciclopedia alfabética. Mérida, Inversiones Cares.

Bingham, Marie Ballew (1972). A Catalog of the Yucatán Collection on Microfilm in the University of Alabama Libraries, Alabama, The University of Alabama Press.

Chartier, Roger (1997). Historia de la lectura en el mundo occidental, Madrid, Taurus.

Chartier, Roger (2016). La mano del autor y el espíritu del impresor, siglos XVI-XVIII, Buenos Aires, KatzEudeba.

Chopin, Alain (2018). "El libro de texto escolar, una falsa evidencia histórica", Revista digital del doctorado en educación de la Universidad Central de Venezuela, vol. 4, núm. 7.

Itinerarios (1851). Itinerarios y leguarios que proceden de Mérida, capital del estado de Yucatán a las vigías de su parte litoral; a las cabeceras de los partidos que la componen; de estas a las que son limítrofes y de los puntos más notables de su costa, s/e

Landa, Diego de (1959). Relación de las cosas de Yucatán, México, Porrúa.

Lanz Cárdenas, Humberto (2008). "Escuela Náutica de Campeche", en Historias dispersas del Instituto Campechano, Campeche, Colección Instituto Campechano.

Lizana, Bernardo de (1988), Historia de Yucatán, Madrid, Historia 16.

López de Cogolludo, Diego (1988), Historia de Yucatán, Campeche, Ayuntamiento de Campeche 3t.

Monreal, Luciana Casilda (1890). Cartilla de higiene y economía doméstica para uso de las escuelas de niñas, Barcelona, Imprenta de Pedro Ortega.

Paz, Octavio (1941). Entre la piedra y la flor. Poemas, México, Nueva Voz.

Ramos i Duarte, Félix (1886). Diccionario de mejicanismos. Colección de locuciones y frases viciosas. Con sus correspondientes críticas i correcciones fundadas en autoridades de la Lengua: máximas, refranes, provincialismos i remoques populares de todos los Estados de la República Mejicana. México, Herrero Hermanos Editores.

Spanish (2020). Spanish First-Hand Accounts on the Battle of St. George's Cay, 1797-1798: Spain's last attempt to occupy Belize. Belize City, University of Belize.

Reed, Nelson A. (2001). The Caste War of Yucatan, Stanford, Stanford University Press.

Viñao, Antonio (2006). "La historia de las disciplinas escolares", Salamanca, Historia de la Educación, núm 25. 